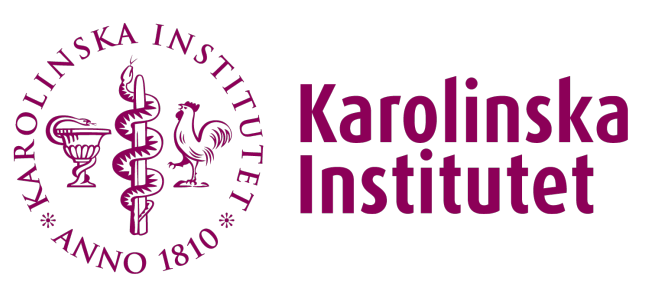

Karolinska Institutet

http://openarchive.ki.se

This is a Peer Reviewed Accepted version of the following article, accepted for publication in Gut.

\title{
Fetal and early life antibiotics exposure and very early onset inflammatory \\ bowel disease : a population-based study
}

Örtqvist, Anne K; Lundholm, Cecilia; Halfvarson, Jonas; Ludvigsson, Jonas F; Almqvist, Catarina

Gut. 2019 Feb;68(2):218-225.

British Society of Gastroenterology

http://doi.org/10.1136/gutjnl-2017-314352

http://hdl.handle.net/10616/46186

If not otherwise stated by the Publisher's Terms and conditions, the manuscript is deposited under the terms of the Creative Commons Attribution-NonCommercial-NoDerivatives License (http://creativecommons.org/licenses/by-nc-nd/4.0/), which permits non-commercial re-use, distribution, and reproduction in any medium, provided the original work is properly cited, and is not altered, transformed, or built upon in any way. 


\section{(19) \\ Karolinska Institutet}

This is an author produced version of a paper accepted by Gut. This paper has been peer-reviewed but does not include the final publisher proof-corrections or journal pagination.

Fetal and early life antibiotics exposure and very early onset inflammatory bowel disease: a population-based study

Örtqvist, Anne K; Lundholm, Cecilia; Halfvarson, Jonas; Ludvigsson, Jonas F; Almqvist, Catarina

Gut : 10 January 2018. [Published Online First]

DOI: $10.1136 /$ gutjnl-2017-314352

Access to the published version may require subscription. Published with permission from: BMJ 
1 Fetal and early life antibiotics exposure and very early onset inflammatory

2 bowel disease - a population-based study

3 Short title: Antibiotics and inflammatory bowel disease

\section{AUTHORS}

6 Anne K. Örtqvist, postdoctoral researcher ${ }^{1}$, Cecilia Lundholm, biostatistician ${ }^{1}$, Jonas Halfvarson,

7 senior lecturer ${ }^{2}$, Jonas F. Ludvigsson, professor ${ }^{1,3}$, Catarina Almqvist, professor ${ }^{1,4}$

9 1. Department of Medical Epidemiology and Biostatistics, Karolinska Institutet, PO Box 281,

$10 \quad 17177$ Stockholm, Sweden

11 2. Department of Gastroenterology, Faculty of Medicine and Health, Örebro University, 70182

12 Örebro, Sweden

13 3. Department of Pediatrics, Örebro University Hospital, 70185 Örebro, Sweden

14 4. Pediatric Allergy and Pulmonology Unit at Astrid Lindgren Children's Hospital, Karolinska

15 University Hospital, 17176 Stockholm, Sweden

\section{ABBREVIATIONS}

19 aHR - adjusted Hazard Ratio

20 CD - Crohn's disease

21 cHR - crude Hazard Ratio

22 CI - Confidence Interval

23 IBD - Inflammatory Bowel Disease

24 MBR - Medical Birth Register

25 NPR - National Patient Register 
$26 \mathrm{PcV}$ - Phenoxymethylpenicillin

27 PIN - Personal Identification Number

28 SPDR - Swedish Prescribed Drug Register

29 TPR - Total Population Register

30 UC - Ulcerative Colitis

31 VEO-IBD - Very Early Onset Inflammatory Bowel Disease

\section{CORRESPONDING AUTHOR}

35 Professor Catarina Almqvist, MD PhD

36 Dept. Medical Epidemiology and Biostatistics

37 PO Box 281, Karolinska Institutet

38 SE 17177 Stockholm, SWEDEN

39 Email. catarina.almqvist@ki.se

$40 \quad$ Telephone. +46701160852

42 KEY WORDS: antibiotics; Crohn's disease; Ulcerative colitis; population-based registers; very

43 early onset (VEO) IBD

44 WORD COUNT: 4309

45 REFERENCES: 56

46 TABLES: 5

47 FIGURES: 2 
51 Objective Earlier studies on antibiotics exposure and development of inflammatory bowel

52 disease (IBD, Crohn's disease and ulcerative colitis) may have been biased by familial factors

53 and gastroenteritis. We aimed to estimate the association between antibiotics during pregnancy or

54 infantile age and very early onset (VEO-) IBD.

55 Design In this cohort study of 827239 children born in Sweden 2006-2013, we examined the link

56 between exposure to systemic antibiotics and VEO-IBD (diagnosis <6 years of age), using Cox

57 proportional hazard regression models. Information on antibiotics and IBD was retrieved from

58 the nationwide population-based Swedish Prescribed Drug Register and the National Patient

59 Register. We specifically examined potential confounding from parental IBD and gastroenteritis.

60 Results Children exposed to antibiotics during pregnancy were at increased risk of IBD

61 compared to general population controls (adjusted hazard ratio (aHR) 1.93; 95\% confidence

62 interval (CI) 1.06-3.50). Corresponding aHRs were 2.48 (1.01-6.08) for Crohn's disease (CD)

63 and $1.25(0.47-3.26)$ for ulcerative colitis (UC) respectively. For antibiotics in infantile age, the

64 aHR for IBD was $1.11(0.57-2.15)$; for CD $0.72(0.27-1.92)$ and $1.23(0.45-3.39)$ for UC.

65 Excluding children with gastroenteritis 12 months prior to the first IBD diagnosis retained similar

66 aHR for antibiotics during pregnancy and CD, while the association no longer remained

67 significant for IBD.

68 Conclusion We found that exposure to antibiotics during pregnancy, but not in infantile age, is

69 associated with an increased risk of VEO-IBD regardless of gastroenteritis. The risk increase for

70 exposure in pregnancy may be due to changes in the microbiota. 


\section{Summary "box"}

73 What is already known about this subject? Very early onset (VEO-) inflammatory bowel

74 disease (IBD) has gradually become more common. One potential risk factor for VEO-IBD is

75 antibiotic exposure during pregnancy and in infantile age.

76 What are the new findings? In this population-based study of more than 800000 children, we

77 found a positive association between antibiotics exposure during pregnancy, but not in infantile

78 age, and later VEO-IBD.

79 How might it impact on clinical practice in the foreseeable future? The risk increase for

80 exposure in pregnancy may be due to changes in the microbiota, which could have an impact on

81 care of pregnant women. However, the absolute risk of disease was very low, and antibiotics

82 during pregnancy should still be used when needed.

83 


\section{INTRODUCTION}

85 Inflammatory bowel disease (IBD), comprising Crohn's disease (CD) and ulcerative colitis (UC),

86 is characterized by chronic inflammation of the gastrointestinal tract. Symptoms related to the

87 disease include diarrhoea, rectal bleeding, abdominal pain and weight loss. Although children

88 (diagnosed at $<18$ years of age) may present with these classical symptoms, non-specific

89 symptoms such as growth failure, anaemia and other extra-intestinal manifestations are also

90 common manifestations of paediatric IBD. ${ }^{1}$ Interestingly, patients with very early onset (VEO)-

91 IBD (defined as diagnosed before six years of age) seem to represent a specific entity, possibly

92 more likely to present with rectal bleeding due to a colonic phenotype (in CD) ${ }^{2}$ and a family

93 history of IBD..$^{2-5}$ This disease entity has gradually become more common and recent data

94 suggest that the increase in IBD incidence is more pronounced in patients with VEO-IBD than

95 among children $\geq 6$ years old, although the overall numbers are still very small compared to

96 young adults. ${ }^{6}$

The pathogenesis is characterized by a complex interaction between genetics, an

98 aberrant mucosal immune response to gut microbiota, disruption of gut barrier, and

99 environmental triggers. While genetic factors clearly play an important role in the etiology of

$100 \mathrm{IBD},{ }^{7-10}$ they cannot explain the recent rise in disease incidence or the proband concordance rate

101 of 38-62\% in monozygotic twins with CD (even less in UC). ${ }^{11} 12$ This has encouraged a search for

102 environmental factors and led to the identification of factors such as smoking, oral contraceptives

103 and appendectomy due to appendicitis (data on appendectomy have however been

104 contradictory) ${ }^{13}$ as potential risk factors in adulthood IBD while less is known for childhood

105 IBD. ${ }^{14-19}$ 
107 remains partly unknown. It has been proposed that the influence of genetics might be more

108 pronounced in VEO-IBD, due to the effect of rare variants with a high penetrance for IBD. ${ }^{20}$

109 However, the role of environmental risk factors in VEO-IBD is largely unknown.

110 One potential risk factor for VEO-IBD is antibiotic exposure during pregnancy and

111 in infantile age. Ungaro et al. reported an increased risk of IBD following antibiotic exposure,

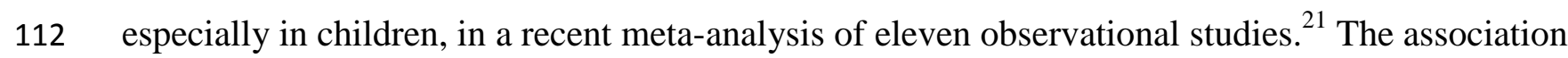

113 was limited to individuals with newly onset CD and not seen for UC. Some earlier studies have

114 also found a positive dose-response relationship between antibiotics exposure and later IBD, but

115 whether this also applies to VEO-IBD remains unknown.

Therefore, we aimed to estimate the association between antibiotics during

117 pregnancy or infantile age and VEO-IBD (CD and UC), while adjusting for parental IBD, as well

118 as taking gastroenteritis and number of doses into account in a population-based study. 


\section{Study population and register linkage}

128 This nationwide prospective population-based register study included all children born January 2006 to December 2013, identified together with their mothers from the Swedish Medical Birth

130 Register (MBR). Fathers to the children were identified through the Swedish Multi-generation 131 Register. $^{22}$ We used the Swedish Prescribed Drug Register (SPDR) ${ }^{23}$ and the National Patient

132 Register (NPR) ${ }^{24}$ to retrieve data on exposure and outcome. The SPDR contains complete data on 133 all dispensed drugs from pharmacies since July 2005 while the NPR began in 1964, became 134 nationwide in 1987 (inpatient diagnoses only), and added hospital-based outpatient visit data in 135 2001. Data from the different registers were linked using the unique personal identity number 136 assigned to all residents in Sweden. ${ }^{25}$

\section{Variables}

138 Antibiotic exposure was defined as filled prescriptions of relevant ATC codes (Anatomical 139 Therapeutic Chemical): J01A-J01X (systemic antibiotics), and were obtained through the SPDR. 140 We categorised antibiotics into two groups: "systemic antibiotics" which included any type of antibiotics; and "PcV" (Phenoxymethylpenicillin), which is by far the most commonly prescribed

142 type of antibiotic in Sweden both during pregnancy and in childhood. ${ }^{26}$

144 according to relevant International Classification of Disease (ICD)-10 codes K50 and K51 145 respectively recorded in the NPR, with onset before 6 years of age. Crohn's disease was defined 146 as $\geq 2 \mathrm{CD}$ diagnoses but never an ulcerative colitis diagnosis and UC was defined as $\geq 2 \mathrm{UC}$ 147 diagnoses but never a CD diagnosis. These outcome definitions have previously been validated 148 by Jakobsson et al. who found a positive predictive value (PPV) of 93\% (95\% CI: 87-97) for 
IBD, 90\% (77-97) for UC and 81\% (67-91) for CD, when compared to the Copenhagen

150 criteria. $^{27}$ While IBD-unclassified is now regarded as a separate entity (and some data suggests

151 that it may represent up to $20 \%$ of the total pediatric IBD population), ${ }^{28}$ this paper focused on CD 152 and UC.

Co-variates; Through the MBR we retrieved information on the child's date of

154 birth, sex, gestational age (days), mode of delivery (vaginal or caesarean section), maternal

155 smoking at first visit to the antenatal care clinic (yes/no), maternal age at delivery $(\leq 19,20-24$,

156 25-29, 30-34, or $\geq 35$ years), and parity (child's birth order at current delivery; first-born (1) or not

157 ( $\geq 2)$ ). Data from Cnattingius et al. suggest a high quality of data in the MBR with a coverage of

$158>98 \%$ of all births in Sweden. ${ }^{29}$

Maternal and paternal IBD was defined from the NPR similarly to that of the

160 children (two records of either: ICD-8: 563.00, 563.10, 569.02; ICD-9: 555, and ICD-10: K50 for

161 CD; and ICD-8: 563.98, 563.99; ICD-9: 556, and ICD-10: K51 for UC). Information on highest

162 level of education of either parent (0-12 vs $>12$ years) was identified through the Longitudinal

163 integration database for health insurance and labour market studies and parents' country of birth

164 (Sweden or other) was identified from the Total Population Register (TPR).

We defined the beginning of the pregnancy (conception date) as date of birth minus gestational age in days. We furthermore divided pregnancy duration into trimester 1 (day 1-91), 2

167 (day 92-189), and 3 (day 190+) to examine time-varying effects of fetal antibiotics exposure. To

168 ensure that the full pregnancy would be covered by this study (especially antibiotics exposure in

169 the first trimester in offspring born close to the starting point of the SPDR), we restricted our

170 study population to children with estimated conception date as on or after July $1^{\text {st }}, 2005$.

171 Information on migration and death was obtained from the TPR to be able to define end of 
172 follow-up. Offspring to women who immigrated to Sweden during pregnancy were excluded 173 from the study $(\mathrm{n}=14030)$.

\section{Statistical analysis}

176 The risk of IBD, and CD and UC separately, in children exposed to antibiotics during pregnancy

177 and in infantile age compared to unexposed children, was examined in Cox proportional hazard

178 models. Attained age was used as the underlying time scale and clustering within families was

179 taken into account by using a sandwich estimator for the standard errors. Follow-up ended with

180 first IBD diagnosis, emigration, death or end of study period (December $31^{\text {st }}, 2014$ i.e at least one

181 year of follow-up), whichever happened first. The proportional hazards assumption was tested

182 using Schoenfeld's residuals ( $p$-values of 0.58 for exposure during pregnancy and 0.84 for

183 exposure in infantile age). Antibiotic exposure during pregnancy was regarded as exposed or non-

184 exposed at the start of follow-up, while exposure after birth was modelled as a time(age)-varying

185 exposure, i.e. a model in which all individuals start as unexposed and then the exposure status

186 changes at the time an individual becomes exposed. However, as there was no sign of non-

187 proportional hazards we did not allow for the effect of the exposure to vary over time.

Potential confounders were identified based on the Directed Acyclic Graphs (DAG)

189 concept, ${ }^{30}$ and the final models were adjusted for: mother's and father's history of IBD, parental

190 education, mother's and father's country of birth (in analyses of exposure during pregnancy and

191 in childhood) and mode of delivery (in analysis of exposure in infantile age) as shown in Figure

192 1. Those with missing information on these variables were excluded $(n=2848$ for exposure in

193 pregnancy and n=3274 for exposure in infantile age) and complete case analyses were performed.

194 Crude and adjusted Hazard Ratios (HR) are presented. 
196 individuals who had been diagnosed with gastroenteritis, either bacterial or viral, according to 197 ICD 10 codes A00-A09, within 12 months prior to onset of their first IBD diagnosis. The risk of 198 IBD with onset after 2 years of age in children exposed to systemic antibiotics in the first year of 199 life, was further tested to investigate potential information bias such as misclassification or 200 reverse causation. This since a possible misclassification of first occurrence of IBD symptoms as 201 gastroenteritis, treated with antibiotics, may induce an association between antibiotics and IBD.

202 A potential dose-relationship between number of antibiotics prescriptions in childhood (1, 2 or $\geq 3$ 203 as a continuous variable) and IBD was tested. Dispensed prescriptions of the same type of 204 antibiotics within 7 days from the prior dispense was counted as one dispense. Too few women 205 had filled more than one prescription of antibiotics during pregnancy to be able to perform dose206 response-analyses. The role of timing of systemic antibiotics exposure during the fetal period $\left(1^{\text {st }}\right.$ 207 vs $2^{\text {nd }}$ vs $3^{\text {rd }}$ trimester) and IBD was further explored. We also examined if the risk increase by 208 antibiotic exposure was dependent on parity by adding interaction terms between parity (first209 born vs. non-first born) and antibiotic exposure.

210 STATA statistical software (version 14) was used for all statistical analyses.

211 The study was approved by the Regional Ethical Review board in Stockholm, Sweden. 


\section{RESULTS}

215 The final study population consisted of 827239 children (Figure 2). Some 12606 children

216 emigrated during follow-up, and another 2428 died.

Overall, $17 \%$ ( $n=140665)$ of the children had been exposed to antibiotics during

218 pregnancy, and 5\% $(n=40116)$ had been exposed on two or more occasions. In infantile age, $65 \%$

$219(\mathrm{n}=539809)$ had been exposed to systemic antibiotics at least once, and 373802 (70\% of those

220 exposed at all) had filled two or more prescriptions (Table 1). 
Table 1. Descriptive table of the study population.

\begin{tabular}{|c|c|c|c|c|c|c|c|c|}
\hline \multirow{3}{*}{ Variable } & \multicolumn{2}{|c|}{ All } & \multicolumn{2}{|c|}{ IBD } & \multicolumn{2}{|c|}{ CD } & \multicolumn{2}{|c|}{$\mathbf{U C}$} \\
\hline & $\mathbf{N}$ & $\%$ & $\mathbf{n}$ & $\%$ & $\mathbf{n}$ & $\%$ & $\mathbf{n}$ & $\%$ \\
\hline & 827239 & 100.0 & 51 & 100.0 & 20 & 100.0 & 24 & 100.0 \\
\hline \multicolumn{9}{|l|}{ Sex } \\
\hline Males & 425212 & 51.4 & 26 & 51.0 & 12 & 60.0 & 11 & 45.8 \\
\hline Females & 402027 & 48.6 & 25 & 49.0 & 8 & 40.0 & 13 & 54.2 \\
\hline $\begin{array}{l}\text { Systemic antibiotic exposure during } \\
\text { pregnancy }\end{array}$ & 140665 & 17.0 & 15 & 29.4 & 7 & 35.0 & 5 & 20.8 \\
\hline Phenoxymethylpenicillin (PcV) & 60701 & 7.3 & 8 & 15.7 & 4 & 20.0 & 3 & 12.5 \\
\hline Pivmecillinam & 34264 & 4.1 & 2 & 3.9 & 1 & 5.0 & 1 & 4.2 \\
\hline Nitrofurantoin & 30904 & 3.7 & 4 & 7.8 & 1 & 5.0 & 2 & 8.3 \\
\hline Other & 42672 & 5.2 & 5 & 9.8 & 4 & 20.0 & 0 & 0 \\
\hline \multicolumn{9}{|l|}{$\begin{array}{l}\text { Number of prescriptions during } \\
\text { pregnancy (any)* }\end{array}$} \\
\hline 1 & 100549 & 12.2 & 10 & 19.6 & 4 & 20.0 & 3 & 12.5 \\
\hline 2 & 26357 & 3.2 & 3 & 5.9 & 2 & 10.0 & 1 & 4.2 \\
\hline$\geq 3$ & 13759 & 1.7 & 2 & 3.9 & 1 & 5.0 & 1 & 4.2 \\
\hline $\begin{array}{l}\text { Systemic antibiotic exposure in } \\
\text { infantile age }\end{array}$ & 539809 & 65.3 & 43 & 84.3 & 16 & 80.0 & 20 & 83.3 \\
\hline Phenoxymethylpenicillin (PcV) & 460283 & 55.6 & 38 & 74.5 & 14 & 70.0 & 17 & 70.8 \\
\hline Amoxicillin & 152052 & 18.4 & 15 & 29.4 & 7 & 35.0 & 5 & 20.8 \\
\hline Flucloxacillin & 80814 & 9.8 & 13 & 25.5 & 5 & 25.0 & 4 & 16.7 \\
\hline Other & 204387 & 24.7 & 28 & 54.9 & 10 & 50.0 & 11 & 45.8 \\
\hline \multicolumn{9}{|l|}{$\begin{array}{l}\text { Number of prescriptions in infantile } \\
\text { age (any)* }\end{array}$} \\
\hline 1 & 166007 & 20.1 & 3 & 5.9 & 1 & 5.0 & 2 & 8.3 \\
\hline 2 & 111761 & 13.5 & 8 & 15.7 & 2 & 10.0 & 6 & 25.0 \\
\hline$\geq 3$ & 262041 & 31.7 & 32 & 62.7 & 13 & 65.0 & 12 & 50.0 \\
\hline \multicolumn{9}{|l|}{ Mothers' diagnoses } \\
\hline$I B D$ & 6780 & 0.8 & 7 & 13.7 & 4 & 20.0 & 2 & 8.3 \\
\hline$C D$ & 1943 & 0.2 & 2 & 3.9 & 2 & 10.0 & 0 & 0.0 \\
\hline$U C$ & 3598 & 0.4 & 5 & 9.8 & 2 & 10.0 & 2 & 8.3 \\
\hline \multicolumn{9}{|l|}{ Fathers' diagnoses } \\
\hline$I B D$ & 7333 & 0.9 & 4 & 7.8 & 0 & 0.0 & 2 & 8.3 \\
\hline$C D$ & 1868 & 0.2 & 0 & 0.0 & 0 & 0.0 & 0 & 0.0 \\
\hline$U C$ & 3972 & 0.5 & 1 & 2.0 & 0 & 0.0 & 0 & 0.0 \\
\hline \multicolumn{9}{|l|}{ Parents' highest achieved education } \\
\hline $0-12$ years & 337312 & 40.8 & 18 & 35.3 & 8 & 40.0 & 7 & 29.2 \\
\hline$>12$ years & 485926 & 58.7 & 33 & 64.7 & 12 & 60.0 & 17 & 70.8 \\
\hline Missing & 4001 & 0.5 & 0 & 0.0 & 0 & 0.0 & 0 & 0.0 \\
\hline \multicolumn{9}{|l|}{ Mother's country of birth } \\
\hline Sweden & 646032 & 78.1 & 42 & 82.4 & 18 & 90.0 & 20 & 83.3 \\
\hline Other & 181207 & 21.9 & 9 & 17.7 & 2 & 10.0 & 4 & 16.7 \\
\hline
\end{tabular}




\begin{tabular}{lcc|cc|cc|cc} 
Sweden & 630372 & 76.2 & 39 & 76.5 & 18 & 90.0 & 19 & 79.2 \\
Other & 178669 & 21.6 & 12 & 23.5 & 2 & 10.0 & 5 & 20.8 \\
Missing & 18198 & 2.2 & 0 & 0.0 & 0 & 0.0 & 0 & 0.0 \\
\hline Mode of delivery & & & & & & & & \\
Vaginal & 679693 & 82.2 & 43 & 84.3 & 16 & 80.0 & 20 & 83.3 \\
$\quad$ Caesarean Section & 147107 & 17.8 & 8 & 15.7 & 4 & 20.0 & 4 & 16.7 \\
Missing & 439 & 0.1 & 0 & 0.0 & 0 & 0.0 & 0 & 0.0 \\
\hline Maternal smoking at first visit to the & & & & & & & & \\
antenatal care clinic & & & & & & & & \\
Yes & 51892 & 6.3 & 3 & 5.9 & 1 & 5.0 & 1 & 4.2 \\
No & 741727 & 89.7 & 46 & 90.2 & 17 & 85.0 & 23 & 95.8 \\
Missing & 33620 & 4.1 & 2 & 3.9 & 2 & 10.0 & 0 & 0.0 \\
\hline Maternal age at delivery (years) & & & & & & & & \\
$\leq 19$ & 12060 & 1.5 & 0 & 0.0 & 0 & 0.0 & 0 & 0.0 \\
$20-24$ & 105879 & 12.8 & 4 & 7.8 & 2 & 10.0 & 1 & 4.2 \\
$25-29$ & 237650 & 28.7 & 18 & 35.3 & 7 & 35.0 & 7 & 29.2 \\
$30-34$ & 287319 & 34.7 & 18 & 35.3 & 5 & 25.0 & 12 & 50.0 \\
$\geq 35$ & 184330 & 22.3 & 11 & 21.6 & 6 & 30.0 & 4 & 16.7 \\
Missing & 1 & 0.0 & 0 & 0.0 & 0 & 0.0 & 0 & 0.0 \\
\hline Parity (at current delivery) & & & & & & & & \\
1 & 361332 & 43.7 & 26 & 51.0 & 11 & 55.0 & 10 & 41.7 \\
$\geq 2$ & 465907 & 56.3 & 25 & 49.0 & 9 & 45.0 & 14 & 58.3 \\
\hline
\end{tabular}

Any - Any type of systemic antibiotics (ATC: J01)

* Independent on time in relation to diagnosis (i.e. either before or after onset of diagnosis)

"Other" antibiotics during pregnancy - Tetracyclines (J01A), penicillins with extended spectrum (J01CA) (except pivmecillinam), beta-lactamase sensitive penicillins (J01CE) (except phenoxymethylpenicillin), beta-lactamase resistant penicillins (J01CF), combinations of penicillins, incl. beta-lactamase inhibitors (J01CR), cephalosporins (J01DB-DD), monobactams (J01DF), carbapenems (J01DH), trimethoprim (J01EA), combinations of sulfonamides and trimethoprim (J01EE), macrolides (J01FA), lincosamides (J01FF), other aminoglycosides (J01GB), fluoroquinolones (J01MA), other antibacterials (J01X) (except nitrofurantoin).

233 "Other" antibiotics during childhood - Tetracyclines (J01A), penicillins with extended spectrum (J01CA) (except amimoxicillin), beta-lactamase resistant penicillins (J01CF) (except flucloxacillin), combinations of penicillins, incl. beta-lactamase inhibitors (J01CR), 236 cephalosporins (J01DB-DE), monobactams (J01DF), carbapenems (J01DH), trimethoprim 237 (J01EA), combinations of sulfonamides and trimethoprim (J01EE), macrolides (J01FA), 238 lincosamides (J01FF), other aminoglycosides (J01GB), fluoroquinolones (J01MA), other 239 antibacterials (J01X). 
242 (interquartile range (IQR) 64-197 days), while the median age at first exposure to systemic 243 antibiotics in childhood was 1.3 years (IQR 0.8-2.1 years).

245 diagnosis) and 24 with UC (but never a CD diagnosis) could be identified through the NPR.

246 Approximately $14 \%$ of the children with IBD had a mother with IBD and $8 \%$ had a father with

247 IBD, compared to those children without IBD, where the corresponding numbers for parental

248 IBD were less than $1 \%(0.8 \%$ mothers and $0.9 \%$ fathers) (Table 1$)$. The median age of the first

249 IBD diagnosis was 2.0 years (IQR 0.9-4.1 years).

\section{Antibiotics during pregnancy}

251 Table 2 presents crude and adjusted Hazard Ratios (aHR) and 95\% Confidence Intervals (CI) for

252 the association between exposure to antibiotics during pregnancy and IBD, CD and UC

253 respectively. There was a 93\% significantly increased risk of IBD in children exposed to systemic

254 antibiotics during pregnancy (aHR1.93, 95\% CI 1.06-3.50). An association remained for CD

255 (aHR 2.48, 95\% CI 1.01-6.08), but not for UC (aHR 1.25, 95\% CI 0.47-3.26). The aHR seemed

256 to remain when restricting systemic antibiotic exposure to PcV (aHR for IBD 2.15, 95\% CI 1.02-

257 4.56), although no longer significant for CD (aHR 2.85, 95\% CI 0.96-8.45). . Still, no significant

258 association was found between "PcV" and UC.

In total, six children had been diagnosed with gastroenteritis 12 months prior to the

260 first diagnosis of IBD, where five had been diagnosed with a viral or unspecified gastroenteritis

261 and colitis (ICD 10: A09) and one had been diagnosed with a bacterial gastroenteritis with

262 Clostridium difficile (ICD 10: A047). In sensitivity analyses, excluding these individuals, similar

263 aHR remained for systemic antibiotics and CD (aHR 2.51, 95\% CI 0.96-6.56), although no longer 
264 significant, and with lower non-significant risk estimates for systemic antibiotics and IBD (aHR $2651.68,95 \%$ CI 0.88-3.21) (Table 2). 
Table 2. Crude and adjusted Hazard ratios and 95\% confidence intervals for inflammatory bowel disease (IBD) in relation to exposure

\begin{tabular}{|c|c|c|c|c|c|c|c|c|c|}
\hline \multirow[b]{2}{*}{ Antibiotics } & \multicolumn{3}{|c|}{ IBD } & \multicolumn{3}{|c|}{ Crohn's disease (CD) } & \multicolumn{3}{|c|}{ Ulcerative colitis (UC) } \\
\hline & $\mathbf{n}$ & cHR $(95 \%$ CI $)$ & aHR $(95 \%$ CI $)$ & $\mathbf{n}$ & cHR $(95 \% \mathrm{CI})$ & aHR $(95 \%$ CI $)$ & $\mathbf{n}$ & cHR $(95 \% \mathrm{CI})$ & aHR $(95 \% \mathrm{CI})$ \\
\hline Any & 15 & $\begin{array}{c}1.96 \\
(1.07-3.57)\end{array}$ & $\begin{array}{c}1.93 \\
(1.06-3.50)\end{array}$ & 7 & $\begin{array}{c}2.52 \\
(1.01-6.28)\end{array}$ & $\begin{array}{c}2.48 \\
(1.01-6.08)\end{array}$ & 5 & $\begin{array}{c}1.24 \\
(0.47-3.32)\end{array}$ & $\begin{array}{c}1.25 \\
(0.47-3.26)\end{array}$ \\
\hline exl.GE & 12 & $\begin{array}{c}1.70 \\
(0.88-3.29)\end{array}$ & $\begin{array}{c}1.68 \\
(0.88-3.21)\end{array}$ & 6 & $\begin{array}{c}2.55 \\
(0.95-6.86)\end{array}$ & $\begin{array}{c}2.51 \\
(0.96-6.56)\end{array}$ & 4 & $\begin{array}{c}1.05 \\
(0.36-3.08)\end{array}$ & $\begin{array}{c}1.05 \\
(0.36-3.08)\end{array}$ \\
\hline$P c V$ & 8 & $\begin{array}{c}2.19 \\
(1.03-4.66)\end{array}$ & $\begin{array}{c}2.15 \\
(1.02-4.56)\end{array}$ & 4 & $\begin{array}{c}2.92 \\
(0.99-8.67)\end{array}$ & $\begin{array}{c}2.85 \\
(0.96-8.45)\end{array}$ & 3 & $\begin{array}{c}1.71 \\
(0.51-5.71)\end{array}$ & $\begin{array}{c}1.68 \\
(0.51-5.97)\end{array}$ \\
\hline exl.GE & 7 & $\begin{array}{c}2.17 \\
(0.97-4.84)\end{array}$ & $\begin{array}{c}2.13 \\
(0.96-4.75) \\
\end{array}$ & 3 & $\begin{array}{c}2.50 \\
(0.73-8.62)\end{array}$ & $\begin{array}{c}2.45 \\
(0.71-8.42) \\
\end{array}$ & 3 & $\begin{array}{c}1.87 \\
(0.56-6.34)\end{array}$ & $\begin{array}{c}1.85 \\
(0.55-6.21)\end{array}$ \\
\hline
\end{tabular}

cHR - Crude Hazard Ratio; 3945000 Person-years

aHR - Adjusted Hazard Ratio; 3874000 Person-years; analyses adjusted for mother's and father's history of IBD, parental education, mother's and father's country of birth. Those with missing information on these variables are excluded (no cases excluded).

$276 P c V-$ Phenoxymethylpenicillin 
278 further explored, where aHR for the first trimester was 1.59 (95\% CI 0.64-3.97), second trimester

$279 \quad 1.23(95 \%$ CI $0.45-3.40)$ and with a significant association during the third trimester (aHR 2.57

$28095 \%$ CI 1.10-6.01) (Table 3). Too few cases were available for separate analyses of exposure in

281 different trimesters and CD/UC.

282 Table 3. Crude and adjusted Hazard ratios and 95\% confidence intervals for the association

283 between systemic antibiotics and inflammatory bowel disease (IBD) in different trimesters during

284 pregnancy.

\begin{tabular}{l|ccc}
\cline { 2 - 4 } \multicolumn{2}{c}{} & \multicolumn{2}{c}{285} \\
\cline { 2 - 4 } Trimester & n & cHR (95\% CI) & aHR (95\% 28. 1 (9) \\
\hline First & 5 & $1.59(0.63-4.01)$ & $1.59(0.64-3.97)$ \\
Second & 4 & $1.27(0.46-3.51)$ & $1.23(0.45-3.40)$ \\
Third & 6 & $2.60(1.11-6.10)$ & $2.57(1.10-6280)$ \\
\hline
\end{tabular}

289 cHR - Crude Hazard Ratio; 3945000 Person-years

290 aHR - Adjusted Hazard Ratio; 3874000 Person-years; analyses adjusted for mother's and 291 father's history of IBD, parental education, mother's and father's country of birth. Those with 292 missing information on these variables are excluded (no cases excluded).

$293 \mathrm{n}$-cases, i.e. those who have been exposed to antibiotics prior to onset of IBD 


\section{Antibiotics in infantile age}

295 Table 4 presents crude and adjusted Hazard Ratios (aHR) and 95\% Confidence Intervals (CI) for 296 the association between exposure to antibiotics in infantile age and IBD, CD and UC

297 respectively. No significant associations were found for systemic antibiotics and "PcV" for IBD,

298 CD or UC, where aHR varied between 0.72 (95\% CI 0.27-1.92) (CD), 1.23 (95\% CI 0.45-3.39)

299 (UC) and 1.11 (95\% CI 0.57-2.15) (IBD) for systemic antibiotics and 0.87 (95\% CI 0.33-2.27)

300 (CD), 1.20 (95\% CI 0.51-2.81) (UC) and 1.25 (95\% CI 0.70-2.26) (IBD) for "PcV". The

301 associations remained non-significant after excluding individuals with gastroenteritis for both

302 systemic antibiotics and "PcV" and all outcomes (IBD, CD and UC). 
Table 4. Crude and adjusted Hazard ratios and 95\% confidence intervals for the association between exposure to systemic antibiotics

\begin{tabular}{|c|c|c|c|c|c|c|c|c|c|}
\hline & \multicolumn{3}{|c|}{ IBD } & \multicolumn{3}{|c|}{ Crohn's disease (CD) } & \multicolumn{3}{|c|}{ Ulcerative colitis (UC) } \\
\hline Antibiotics & n & cHR $(95 \%$ CI $)$ & aHR $(95 \%$ CI) & $\mathbf{n}$ & cHR $(95 \%$ CI $)$ & aHR $(95 \%$ CI $)$ & $\mathbf{n}$ & cHR $(95 \% \mathrm{CI})$ & aHR $(95 \%$ CI $)$ \\
\hline Any & 25 & $\begin{array}{c}1.13 \\
(0.58-2.20)\end{array}$ & $\begin{array}{c}1.11 \\
(0.57-2.15)\end{array}$ & 9 & $\begin{array}{c}0.72 \\
(0.27-1.94)\end{array}$ & $\begin{array}{c}0.72 \\
(0.27-1.92)\end{array}$ & 11 & $\begin{array}{c}1.22 \\
(0.44-3.37)\end{array}$ & $\begin{array}{c}1.23 \\
(0.45-3.39)\end{array}$ \\
\hline exl.GE & 23 & $\begin{array}{c}1.28 \\
(0.60-2.73)\end{array}$ & $\begin{array}{c}1.26 \\
(0.60-2.64)\end{array}$ & 7 & $\begin{array}{c}0.66 \\
(0.22-1.99)\end{array}$ & $\begin{array}{c}0.66 \\
(0.23-1.92)\end{array}$ & 11 & $\begin{array}{c}1.39 \\
(0.46-4.25)\end{array}$ & $\begin{array}{c}1.40 \\
(0.46-4.25)\end{array}$ \\
\hline$P c V$ & 22 & $\begin{array}{c}1.27 \\
(0.70-2.31)\end{array}$ & $\begin{array}{c}1.25 \\
(0.70-2.26)\end{array}$ & 8 & $\begin{array}{c}0.88 \\
(0.33-2.31)\end{array}$ & $\begin{array}{c}0.87 \\
(0.33-2.27)\end{array}$ & 9 & $\begin{array}{c}1.19 \\
(0.50-2.80)\end{array}$ & $\begin{array}{c}1.20 \\
(0.51-2.81)\end{array}$ \\
\hline exl.GE & 21 & $\begin{array}{c}1.55 \\
(0.80-3.00)\end{array}$ & $\begin{array}{c}1.52 \\
(0.80-2.90)\end{array}$ & 7 & $\begin{array}{c}1.07 \\
(0.36-3.21)\end{array}$ & $\begin{array}{c}1.06 \\
(0.37-3.10)\end{array}$ & 9 & $\begin{array}{c}1.29 \\
(0.52-3.23)\end{array}$ & $\begin{array}{c}1.31 \\
(0.53-3.22)\end{array}$ \\
\hline
\end{tabular}

306

cHR - Crude Hazard Ratio; 3945000 Person-years

aHR - Adjusted Hazard Ratio; 3874000 Person-years; analyses adjusted for mother's and father's history of IBD, parental education, mother's and father's country of birth and mode of delivery. Those with missing information on these variables are excluded (no cases excluded).

$\mathrm{n}$ - cases, i.e. those who have been exposed to antibiotics prior to onset of IBD/UC/CD diagnosis

exl.GE - excluding gastroenteritis, either bacterial or viral, according to ICD 10 codes A00-A09 12 months prior to onset of the first IBD diagnosis.

$P c V-$ Phenoxymethylpenicillin 
316 antibiotics during the first year of life and the risk of first IBD diagnosis from 2 years of age, the

317 aHR was 1.49 (95\% CI 0.69-3.22). Furthermore, no significant interaction $(p=0.48)$ was found

318 between systemic antibiotics and parity and IBD, where the aHR in first-born was 0.93 (95\% CI

321 prescriptions for systemic antibiotics in infantile age and IBD (Table 5).

322 Table 5. Crude and adjusted Hazard ratios and 95\% confidence intervals for inflammatory bowel

323 disease (IBD) in relation to increasing number of antibiotic prescriptions.

\begin{tabular}{l|lrr}
\cline { 2 - 4 } \multicolumn{2}{c}{} & \multicolumn{2}{c}{ IBD } \\
\hline $\begin{array}{l}\text { Systemic antibiotics, } \\
\text { filled prescriptions }\end{array}$ & n & cHR (95\% CI) & aHR (95\% CI) \\
\hline 1 & 6 & $0.73(0.28-1.92)$ & $0.73(0.28-1.89)$ \\
2 & 9 & $1.87(0.81-4.34)$ & $1.85(0.80-4.30)$ \\
$\geq 3$ & 10 & $1.15(0.48-2.75)$ & $1.12(0.47-2.325)$ \\
\hline
\end{tabular}

328 cHR - Crude Hazard Ratio; 3945000 Person-years

329 aHR - Adjusted Hazard Ratio; 3874000 Person-years; analyses adjusted for mother's and 330 father's history of IBD, parental education, mother's and father's country of birth and mode of 331 delivery. Those with missing information on these variables are excluded (no cases excluded).

$332 \mathrm{n}$-cases, i.e. those who have been exposed to antibiotics prior to onset of IBD diagnosis 
336 In this nationwide population-based birth cohort study of more than 800000 children, we found a 337 positive association between antibiotics exposure during pregnancy and later VEO-IBD and CD, 338 but not UC. The aHR seemed to remain when restricting systemic antibiotic exposure to PcV, 339 although no longer significant for CD. The risk estimates for systemic antibiotics and CD 340 remained similar after exclusion of children with gastroenteritis 12 months prior to their first IBD

341 diagnosis. No association was found between systemic antibiotics or "PcV" in infantile age and 342 later VEO-IBD, CD or UC, independent of exclusion of individuals with gastroenteritis prior to 343 their first IBD diagnosis. This is important as gastroenteritis may have represented undiagnosed 344 IBD, and resulted in antibiotics treatment occurring after IBD rather than preceding it.

345 Furthermore, there was no significant association for children who had been exposed to systemic 346 antibiotics during the first year of life and the risk of first IBD diagnosis from 2 years of age, or 347 between increasing numbers of filled prescriptions for antibiotics and IBD.

\section{$348 \quad$ Previous literature}

349 While recent research has confirmed that antibiotic use is associated with an increased risk of 350 IBD, including pediatric CD, less attention has been paid to VEO-IBD. In a recent meta-analysis, 351 Ungaro et al. examined 11 studies, ${ }^{31-41}$ with four focusing on paediatric IBD. ${ }^{3437} 3940$ The authors 352 regarded only three of these as paediatric, ${ }^{343739}$ and when pooling their data the HR was 353 substantially higher than in the overall meta-analysis (HR 2.75, 95\% CI 1.72-4.38) for children 354 only). antibiotics may be causally related to IBD, potentially by a mediating effect on the microbiome,

357 causing a reduced diversity and an increased dysbiosis. ${ }^{42}$ The microbiome interacts with the host 
358 through production of short-chain fatty acids (including butyrate), induction of the mucosal

359 immune system, stimulation of the local nervous system but also through interaction with the 360 lamina propria by modification of the gut barrier function. ${ }^{43}$ A dysbiosis in the gut microbiota,

361 characterized by reduction of beneficial bacteria such as Faecalibacterium prausnitzii, and

362 Ruminococcaceae and an increase of pathogens or pathobionts, has consistently been shown in

363 patients with IBD, especially ileal CD. ${ }^{44}$ Compared to previous studies we did not find any

364 association between antibiotics treatment in infantile age and IBD, maybe due to that VEO-IBD

365 is partly a different entity from later-onset IBD. ${ }^{31} 39$ On the other hand, we found a two-fold

366 increased risk in offspring to mothers receiving antibiotics during pregnancy. We suggest such

367 antibiotics exposure may be detrimental to the risk of VEO-IBD in the offspring. The gut

368 microbiota in pregnant women resembles that of healthy non-pregnant women during the first

369 two trimesters, but undergoes substantial changes during the third trimester. ${ }^{45}$ In our study, the

370 highest risk of later VEO-IBD was seen in mothers exposed to antibiotics in the last trimester

371 (aHR 2.57 95\% CI 1.10-6.01), i.e. just before birth. Recent animal research indicates that

372 antibiotics administered during pregnancy have substantial effects on the offspring microbiome

373 (reduced bacterial diversity), but may also influence the immune response in the offspring ${ }^{45}$ and

374 increase susceptibility to develop colonic inflammation. ${ }^{46}$

While it has long been thought that the intestinal tract is sterile at birth, recent data

376 suggest that the microbial colonization process may be initiated already in utero, ${ }^{47}$ a process that

377 may be affected by antibiotics late in pregnancy. Furthermore, it has been suggested in studies of

378 repeated fecal samples from term infants that the use of intrapartum PcV prophylaxis, to prevent

379 early onset group B streptococcal infection in newborns, alters the offspring microbiome, ${ }^{48} 49$

380 although others have found very few differences between antibiotic-exposed and non-antibiotic- 
exposed infants. ${ }^{50}$ Meanwhile maternal intake of probiotics influence the expression of toll-like

382 receptors in infant meconium, ${ }^{51}$ indicating that fetal exposure of antibiotics may play an

383 important role for the development microbiota and the immune system of the child. ${ }^{48}$ While a

384 causative association between antibiotics during pregnancy and VEO-IBD thus seems plausible,

385 we cannot exclude possible confounding from an intrauterine infection with fever of the mother

386 and a raised inflammatory reaction, causing epigenetic imprinting in the fetus and subsequent

387 VEO-IBD. Bernstein and colleagues recently suggested that individuals with IBD were no more

388 likely than controls to have been born to mothers with peripartum infections, however they were

389 diagnosed at an earlier age than those whose mothers did not have an infection. ${ }^{52}$ Unfortunately,

390 we had no data on the indications of the mother's antibiotic treatment during pregnancy or on

391 either symptoms (or date of symptom onset) or genotype in patients, neither were we able to

392 examine the microbiota per se in children with VEO-IBD

In the meta-analysis by Ungaro et al. also fluoroqinolones were highly linked to

394 IBD (pooled OR=1.79, 95\% CI 1.03-3.12). ${ }^{21}$ During the study period fluoroqinolones were not

395 recommended for younger children in Sweden and only accounted for $0.2 \%$ of all antibiotic

396 prescriptions, we therefore chose not to examine fluoroqinolone exposure and IBD separately.

397 The fact that the highest risk estimates for IBD have previously been shown for metronidazole ${ }^{21}$

$398{ }^{31}$ and fluoroqinolones ${ }^{21}$ (both used in the treatment of IBD, although not currently

399 recommended ${ }^{53}$ ) suggest that reverse causation may have been an issue in earlier studies

400 demonstrating an positive association between antibiotics treatment and later IBD. ${ }^{31}{ }^{39} \mathrm{We}$ did not

401 find any association for IBD when we restricted our analysis to antibiotics in the first year of life

402 and our outcome to IBD onset beyond 2 years of age. That sub-analysis is similar to the analysis

403 by Ungaro et al. ${ }^{21}$ limiting their dataset to studies with $\geq 1$ year of exclusion time between 
antibiotics exposure and IBD (HR 1.50, 95\% CI 1.44-1.57), while we found an aHR of 1.49 with

405 broad confidence intervals (95\% CI 0.69-3.22). Reverse causation is obviously not a concern

406 regarding the possible link between antibiotics exposure during pregnancy and risk of VEO-IBD

407 in the offspring.

\section{Strengths and limitations}

409 The main strength of this study is the nationwide cohort, based on prospective information

410 retrieved from high quality population-based register, thereby eliminating recall bias. In addition,

411 we were able to carry out important sub-analyses such as exclusion of individuals with

412 gastroenteritis prior to their first IBD diagnosis, as well as including a time window between

413 exposure and outcome, analyses that allow us to study the potential influence by information bias.

414 Furthermore, we were able to consider familial factors such as parental history of IBD, parents'

415 country of birth and socioeconomic factors including education level.

We acknowledge a number of limitations. Despite our use of a nationwide register-

417 based cohort of more than 800000 children, we could only identify 51 cases of IBD in total.

418 Thus, the number of individuals in each subgroup (CD, UC) was quite low, which is reflected by

419 the rather wide confidence intervals and may also be of concern in the adjusted analyses, however

420 crude and adjusted estimates were very similar. Furthermore, we acknowledge the potential

421 difficulties to diagnose UC in this age group, why stratification of patients into UC versus CD

422 may not be completely accurate. This means that even a small number of misclassified patients

423 may have affected our conclusions, since some of the results are of borderline significance,

424 specifically in the sensitivity analyses where we excluded individuals whose potential first

425 episode of IBD could have been misclassified as gastroenteritis. Nevertheless, we believe that the

426 strength of our data lies in the results seen in IBD in general, which is not dependent on 
stratification into UC versus CD. Misclassification of a child's IBD diagnosis after maternal

428 exposure to antibiotics in pregnancy is most likely non-differential, whereas it could be

429 differential for exposure in infantile age. The misclassification of father's diagnoses related to

430 maternal exposure during pregnancy and to exposure in infantile age is most likely non-

431 differential, whereas misclassification of mother's diagnoses after exposure during pregnancy

432 could be differential, but most likely non-differential for exposure in infantile age. A non-

433 differential misclassification of the outcome will generally bias towards the null, whereas

434 differential misclassification could lead to both higher and lower risk estimates.

Overall, the limited follow-up time of our study means that we were unable to

436 examine the long-term effect of fetal and early life antibiotics on IBD in adulthood, which may

437 also be one possible explanation to the low number of identified cases and lack of association

438 between antibiotic exposure in childhood and later IBD. While a previous validation of IBD,

439 using the same definition (requiring $\geq 2$ diagnoses of IBD) found a positive predictive value

440 (PPV) of 93\%, ${ }^{27}$ we acknowledge that this PPV was calculated in a population of a much higher

441 median age and we cannot rule out that the PPV for $\geq 2$ IBD records in young age is different. As

442 data from primary out-patient care are not available in the NPR, the sensitivity of our study could

443 be lower than in real life. However, we believe that the sensitivity of IBD in the NPR is high for

444 children as paediatric IBD patients are managed by hospital-based specialists and closely

445 monitored with visits every three to six months. ${ }^{28}$ In addition, to minimize the risk of false

446 positive cases and to increase the specificity, we used $\geq 2$ diagnoses for our outcome, even though

447 this could mean that we probably excluded some true cases with a lower sensitivity as result. The

448 small number of cases also limited our possibility to perform sibling analysis, which otherwise

449 would have helped us to control for all factors siblings share (both genetic and environmental). 
451 are available in Sweden today. We have previously shown that $13 \%$ of vaginal deliveries are

452 associated with intrapartum exposure to antibiotics. ${ }^{54}$ The corresponding number for elective 453 caesarean section was $14 \%$, and $63 \%$ for emergency caesarean section. Thus, while the majority

454 of antibiotics in Sweden $(87 \%)$ are prescribed in out-patient care ${ }^{55}$, we did not capture those

455 exposed to antibiotics during the immediate intrapartum period, but were able to adjust for mode

456 of delivery as a co-variate. Exposure of antibiotics was furthermore defined as having filled a

457 prescription of antibiotics, which is not equivalent to adherence to treatment. ${ }^{56}$ Finally, the

458 number of children receiving different subtypes of antibiotics was limited, wherefore we were

459 only able to perform stratified analyses on $\mathrm{PcV}$.

460 Conclusion and implications

461 In conclusion, we found an association between antibiotics exposure during pregnancy,

462 specifically during the third trimester, but not in infantile age, and subsequent development of

463 VEO-IBD. Our results may indicate that antibiotic exposure in late pregnancy can lead to

464 changes in the microbiome of the child, however further research is needed to confirm our

465 findings. In addition, the absolute risk of VEO-IBD is very low, and antibiotics during pregnancy

466 should still be used when needed.

\section{ACKNOWLEDGEMENTS}

469 We direct our great appreciation to Christina Norrby and Marcus Boman who contributed with 470 excellent data collection and management. 


\section{COMPETING INTERESTS}

474 All authors have completed the ICMJE uniform disclosure form at

475 www.icmje.org/coi_disclosure.pdf. AKÖ, CL, JH, JFL and CA claim no conflict of interest

476 related to the submitted work.

477

478

479

480

481

482

483

484

485

486

487

488

489

490

491

492

493

494

495

\section{FUNDING}

Financial support was provided from the Swedish Research Council through the Swedish Initiative for Research on Microdata in the Social And Medical Sciences (SIMSAM) framework grant no 340-2013-5867, grants provided by the Stockholm County Council (ALF-projects), the Swedish Heart-Lung Foundation and the Swedish Asthma and Allergy Association's Research Foundation.

\section{AUTHOR CONTRIBUTIONS}

The study was initiated by JFL and CA, and designed by AKÖ, CL, JFL and CA. AKÖ and CL performed the statistical analysis and wrote the initial draft together with JFL, JH and CA. All authors contributed with invaluable support for data analyses, interpretation of findings and critical revision of the article. CA obtained the financial support. All authors had full access to data, reviewed and approved the final version of the article submitted for publication. AKÖ, CL, JFL, JH and CA are the guarantors for the study and accept full responsibility for the work, had access to the data, and controlled the decision to publish. 


\section{REFERENCES}

497

498

499

500

501

502

503

504

505

506

507

508

509

510

511

512

513

514

515

516

517

518

1. Rosen MJ, Dhawan A, Saeed SA. Inflammatory Bowel Disease in Children and Adolescents. JAMA pediatrics 2015;169(11):1053-60.

2. Gupta N, Bostrom AG, Kirschner BS, et al. Presentation and disease course in early- compared to later-onset pediatric Crohn's disease. The American journal of gastroenterology 2008;103(8):2092-8.

3. Paul T, Birnbaum A, Pal DK, et al. Distinct phenotype of early childhood inflammatory bowel disease. Journal of clinical gastroenterology 2006;40(7):583-6.

4. Heyman MB, Kirschner BS, Gold BD, et al. Children with early-onset inflammatory bowel disease (IBD): analysis of a pediatric IBD consortium registry. The Journal of pediatrics 2005;146(1):35-40.

5. Bequet E, Sarter H, Fumery M, et al. Incidence and Phenotype at Diagnosis of Very-earlyonset Compared with Later-onset Paediatric Inflammatory Bowel Disease: A Populationbased Study [1988-2011]. Journal of Crohn's \& colitis 2017;11(5):519-26.

6. Benchimol EI, Mack DR, Nguyen GC, et al. Incidence, outcomes, and health services burden of very early onset inflammatory bowel disease. Gastroenterology 2014;147(4):803-13.e7; quiz e14-5.

7. Satsangi J, Parkes M, Louis E, et al. Two stage genome-wide search in inflammatory bowel disease provides evidence for susceptibility loci on chromosomes 3, 7 and 12. Nat Genet 1996;14(2):199-202.

8. Hampe J, Cuthbert A, Croucher PJ, et al. Association between insertion mutation in NOD2 gene and Crohn's disease in German and British populations. Lancet 2001;357(9272):1925-8. 
519

520

521

522

523

524

525

526

527

528

529

530

531

532

533

534

535

536

537

538

539

540

541

542

9. Ogura Y, Bonen DK, Inohara N, et al. A frameshift mutation in NOD2 associated with susceptibility to Crohn's disease. Nature 2001;411(6837):603-6.

10. Jostins L, Ripke S, Weersma RK, et al. Host-microbe interactions have shaped the genetic architecture of inflammatory bowel disease. Nature 2012;491(7422):119-24.

11. Ek WE, D'Amato M, Halfvarson J. The history of genetics in inflammatory bowel disease. Annals of gastroenterology : quarterly publication of the Hellenic Society of Gastroenterology 2014;27(4):294-303.

12. Zhulina Y, Hahn-Stromberg V, Shamikh A, et al. Subclinical inflammation with increased neutrophil activity in healthy twin siblings reflect environmental influence in the pathogenesis of inflammatory bowel disease. Inflammatory bowel diseases 2013;19(8):1725-31.

13. Parian A, Limketkai B, Koh J, et al. Appendectomy does not decrease the risk of future colectomy in UC: results from a large cohort and meta-analysis. Gut 2017;66(8):1390-97.

14. Harries AD, Baird A, Rhodes J. Non-smoking: a feature of ulcerative colitis. Br Med J (Clin Res Ed) 1982;284(6317):706.

15. Tysk C, Lindberg E, Jarnerot G, et al. Ulcerative colitis and Crohn's disease in an unselected population of monozygotic and dizygotic twins. A study of heritability and the influence of smoking. Gut 1988;29(7):990-6.

16. Andersson RE, Olaison G, Tysk C, et al. Appendectomy is followed by increased risk of Crohn's disease. Gastroenterology 2003;124(1):40-6.

17. Andersson RE, Olaison G, Tysk C, et al. Appendectomy and protection against ulcerative colitis. N Engl J Med 2001;344(11):808-14.

18. Radford-Smith GL, Edwards JE, Purdie DM, et al. Protective role of appendicectomy on onset and severity of ulcerative colitis and Crohn's disease. Gut 2002;51(6):808-13. 
543

544

545

546

547

548

549

550

551

552

553

554

555

556

557

558

559

560

561

562

563

564

565

19. Khalili H, Granath F, Smedby KE, et al. Association Between Long-term Oral Contraceptive Use and Risk of Crohn's Disease Complications in a Nationwide Study. Gastroenterology 2016;150(7):1561-67.e1.

20. Snapper SB. Very-Early-Onset Inflammatory Bowel Disease. Gastroenterology \& hepatology 2015;11(8):554-6.

21. Ungaro R, Bernstein CN, Gearry R, et al. Antibiotics associated with increased risk of newonset Crohn's disease but not ulcerative colitis: a meta-analysis. The American journal of gastroenterology 2014;109(11):1728-38.

22. Ekbom A. The Swedish Multi-generation Register. Methods Mol Biol 2011;675:215-20.

23. Wettermark B, Hammar N, Fored CM, et al. The new Swedish Prescribed Drug Register-opportunities for pharmacoepidemiological research and experience from the first six months. Pharmacoepidemiol Drug Saf 2007;16(7):726-35.

24. Ludvigsson JF, Andersson E, Ekbom A, et al. External review and validation of the Swedish national inpatient register. BMC Public Health 2011;11(1):450.

25. Ludvigsson JF, Otterblad-Olausson P, Pettersson BU, et al. The Swedish personal identity number: possibilities and pitfalls in healthcare and medical research. European journal of epidemiology 2009;24(11):659-67.

26. Adriaenssens N, Coenen S, Versporten A, et al. European Surveillance of Antimicrobial Consumption (ESAC): outpatient antibiotic use in Europe (1997-2009). J Antimicrob Chemother 2011;66 Suppl 6:vi3-12.

27. Jakobsson GL, Sternegard E, Olen O, et al. Validating inflammatory bowel disease (IBD) in the Swedish National Patient Register and the Swedish Quality Register for IBD (SWIBREG). Scandinavian journal of gastroenterology 2017;52(2):216-21. 
28. Ludvigsson JF, Busch K, Olen O, et al. Prevalence of paediatric inflammatory bowel disease in Sweden: a nationwide population-based register study. BMC gastroenterology 2017;17(1):23.

29. Cnattingius S, Ericson A, Gunnarskog J, et al. A quality study of a medical birth registry. Scand J Soc Med 1990;18(2):143-8.

30. Greenland S, Pearl J, Robins JM. Causal diagrams for epidemiologic research. Epidemiology (Cambridge, Mass) 1999;10(1):37-48.

31. Card T, Logan RF, Rodrigues LC, et al. Antibiotic use and the development of Crohn's disease. Gut 2004;53(2):246-50.

32. Van Kruiningen HJ, Joossens M, Vermeire S, et al. Environmental factors in familial Crohn's disease in Belgium. Inflammatory bowel diseases 2005;11(4):360-5.

33. Gearry RB, Richardson AK, Frampton CM, et al. Population-based cases control study of inflammatory bowel disease risk factors. Journal of gastroenterology and hepatology 2010;25(2):325-33.

34. Shaw SY, Blanchard JF, Bernstein CN. Association between the use of antibiotics in the first year of life and pediatric inflammatory bowel disease. The American journal of gastroenterology 2010;105(12):2687-92.

35. Han DY, Fraser AG, Dryland P, et al. Environmental factors in the development of chronic inflammation: a case-control study on risk factors for Crohn's disease within New Zealand. Mutation research 2010;690(1-2):116-22.

36. Castiglione F, Diaferia M, Morace F, et al. Risk factors for inflammatory bowel diseases according to the "hygiene hypothesis": a case-control, multi-centre, prospective study in Southern Italy. Journal of Crohn's \& colitis 2012;6(3):324-9. 
589

590

591

592

593

594

595

596

597

598

599

600

601

602

603

604

605

606

607

608

609

610

611

612

37. Virta L, Auvinen A, Helenius H, et al. Association of repeated exposure to antibiotics with the development of pediatric Crohn's disease--a nationwide, register-based finnish casecontrol study. American journal of epidemiology 2012;175(8):775-84.

38. Margolis DJ, Fanelli M, Hoffstad O, et al. Potential association between the oral tetracycline class of antimicrobials used to treat acne and inflammatory bowel disease. The American journal of gastroenterology 2010;105(12):2610-6.

39. Hviid A, Svanstrom H, Frisch M. Antibiotic use and inflammatory bowel diseases in childhood. Gut 2011;60(1):49-54.

40. Kronman MP, Zaoutis TE, Haynes K, et al. Antibiotic exposure and IBD development among children: a population-based cohort study. Pediatrics 2012;130(4):e794-803.

41. Shaw SY, Blanchard JF, Bernstein CN. Association between the use of antibiotics and new diagnoses of Crohn's disease and ulcerative colitis. The American journal of gastroenterology 2011;106(12):2133-42.

42. Gevers D, Kugathasan S, Denson LA, et al. The treatment-naive microbiome in new-onset Crohn's disease. Cell host \& microbe 2014;15(3):382-92.

43. Imhann F, Vich Vila A, Bonder MJ, et al. Interplay of host genetics and gut microbiota underlying the onset and clinical presentation of inflammatory bowel disease. Gut 2016.

44. Halfvarson J, Brislawn CJ, Lamendella R, et al. Dynamics of the human gut microbiome in inflammatory bowel disease. Nature microbiology 2017;2:17004.

45. Koren O, Goodrich JK, Cullender TC, et al. Host remodeling of the gut microbiome and metabolic changes during pregnancy. Cell 2012;150(3):470-80.

46. Munyaka PM, Eissa N, Bernstein CN, et al. Antepartum Antibiotic Treatment Increases Offspring Susceptibility to Experimental Colitis: A Role of the Gut Microbiota. PloS one 2015;10(11):e0142536. 
613

614

615

616

617

618

619

620

621

622

623

624

625

626

627

628

629

630

631

632

633

634

635

47. Collado MC, Rautava S, Aakko J, et al. Human gut colonisation may be initiated in utero by distinct microbial communities in the placenta and amniotic fluid. Scientific reports 2016;6:23129.

48. Nogacka A, Salazar N, Suarez M, et al. Impact of intrapartum antimicrobial prophylaxis upon the intestinal microbiota and the prevalence of antibiotic resistance genes in vaginally delivered full-term neonates. Microbiome 2017;5(1):93.

49. Azad MB, Konya T, Persaud RR, et al. Impact of maternal intrapartum antibiotics, method of birth and breastfeeding on gut microbiota during the first year of life: a prospective cohort study. BJOG : an international journal of obstetrics and gynaecology 2016;123(6):983-93.

50. Jaureguy F, Carton M, Panel P, et al. Effects of intrapartum penicillin prophylaxis on intestinal bacterial colonization in infants. Journal of clinical microbiology 2004;42(11):5184-8.

51. Rautava S, Collado MC, Salminen S, et al. Probiotics modulate host-microbe interaction in the placenta and fetal gut: a randomized, double-blind, placebo-controlled trial. Neonatology 2012;102(3):178-84.

52. Bernstein CN, Burchill C, Targownik LE, et al. Maternal Infections That Would Warrant Antibiotic Use Antepartum or Peripartum Are Not a Risk Factor for the Development of IBD: A Population-Based Analysis. Inflammatory bowel diseases 2017;23(4):635-40.

53. Gomollon F, Dignass A, Annese V, et al. 3rd European Evidence-based Consensus on the Diagnosis and Management of Crohn's Disease 2016: Part 1: Diagnosis and Medical Management. Journal of Crohn's \& colitis 2017;11(1):3-25.

54. Ahlen KM, Ortqvist AK, Gong T, et al. Antibiotic Treatment and Length of Hospital Stay in Relation to Delivery Mode and Prematurity. PloS one 2016;11(10):e0164126. 
636 55. Hellman J O-LB, Bengtsson B, Greko C. SWEDRES-SVARM 2016. Use of antimicrobials

637 and occurrence of antimicrobial resistance in Sweden Secondary SWEDRES-SVARM

638 2016. Use of antimicrobials and occurrence of antimicrobial resistance in Sweden

639 http://www.sva.se/globalassets/redesign2011/pdf/om_sva/publikationer/swedres_svarm20

$640 \quad \underline{16 . p d f}$.

641 56. Kardas P. Patient compliance with antibiotic treatment for respiratory tract infections. J

$642 \quad$ Antimicrob Chemother 2002;49(6):897-903.

643

644 


\section{Figure legends and footnotes for figures}

646 Figure 1. A Directed Acyclic Graph (DAG) depicting the included variables in the final models. 647

648

649 Footnote:

650 The DAG can be applied to various analyses, for example, a study of the association between 651 antibiotic exposure and VEO-IBD. A directed arrow between these two variables indicates that 652 the exposure is associated with the outcome. DAGs can be used to identify all potential 653 confounders that may exist, in order to know which variables that should be adjusted for in the 654 analyses. When the exposure and the outcome are common causes for a third factor (a common 655 effect), this factor is called a collider. Pathways through colliders are closed, unless the collider 656 is adjusted for which will then open the path and potentially cause spurious associations. In this 657 DAG, parental education is a potential collider through parental IBD, however, with adjustment 658 of parental IBD, this backdoor pathway is closed.

$659 *$ Mode of delivery was only included in analysis of antibiotic exposure in infantile age. 660 661 662 663 
664 Figure 2. Flow chart of final study population.

665

666 Footnote:

667 *The first estimated conception date, based on date of birth and gestational age in days, is July 1,

668 2005, and the first child in the cohort was born January 8, 2006. The last estimated conception

669 date was July 7, 2013, and the child was born after 164 days on December 30, 2013.

670 MBR - Medical Birth Register

671 MGR - Multi-generation Register

672 SPDR - Swedish Prescribed Drug Register

673 LISA - Longitudinal integration database for health insurance and labour market studies

674 TPR - Total Population Register

675

676 